\title{
Jane Taylor, The Making of Poetry. Late-Medieval French Poetic Anthologies
}

\section{Paola Cifarelli}

\section{(2) OpenEdition}

1 Journals

\section{Edizione digitale}

URL: http://journals.openedition.org/studifrancesi/8834

DOI: ERREUR PDO dans/localdata/www-bin/Core/Core/Db/Db.class.php L.34 : SQLSTATE[HYO00]

[2006] MySQL server has gone away

ISSN: 2421-5856

\section{Editore}

Rosenberg \& Sellier

\section{Edizione cartacea}

Data di pubblicazione: 1 octobre 2008

Paginazione: 433-434

ISSN: 0039-2944

Notizia bibliografica digitale

Paola Cifarelli, «Jane Taylor, The Making of Poetry. Late-Medieval French Poetic Anthologies», Studi Francesi [Online], 155 (LII | II) | 2008, online dal 30 novembre 2015, consultato il 08 janvier 2021. URL: http://journals.openedition.org/studifrancesi/8834 ; DOI: https://doi.org/10.4000/studifrancesi.8834

Questo documento è stato generato automaticamente il 8 janvier 2021.

\section{(c) $($ ) $\odot$}

Studi Francesi è distribuita con Licenza Creative Commons Attribuzione - Non commerciale - Non opere derivate 4.0 Internazionale. 


\title{
Jane Taylor, The Making of Poetry. Late-Medieval French Poetic Anthologies
}

\author{
Paola Cifarelli
}

\section{NOTIZIA}

JANE TAYLOR, The Making of Poetry. Late-Medieval French Poetic Anthologies, Turnhout, Brepols, 2007 («Texts and Transitions: Studies in the History of Manuscripts and Printed Books», 1), pp. 310.

1 Questo interessante volume è dedicato alle raccolte di poesia lirica apparse in Francia, manoscritte o a stampa, durante il Quattrocento e nei primissimi anni del Cinquecento. Alcuni dei codici presi in esame sono molto noti ed anche molto studiati, come il celebre codice BnF, fr. 25458, che contiene l'antologia poetica di Charles d'Orléans, o l'Harley 4431 della British Library, in cui Christine de Pizan raccolse le sue liriche per presentarle a Isabella di Baviera. Altri sono stati invece spesso trascurati dalla critica, che li ha per lo più etichettati come monotoni e poco originali: è il caso dell'antologia raccolta nel ms. 375 della Bibliothèque Inguimbertine di Carpentras o di quella conservata nel ms. fr. 9223 della BnF, o ancora del monumentale volume a stampa intitolato Jardin de Plaisance, pubblicato da Antoine Vérard. L'elemento che accomuna tutti i testi analizzati, siano essi antologie (ovvero volumi che inseriscono testi diversi sulla base di un principio organizzatore, cf. p. 231) o miscellanee (risultato di inclusioni più casuali), è il fatto che essi siano innanzi tutto il frutto di uno scambio sociale tra individui, di un esercizio conviviale. Questo punto di vista, adottato dall'A. nella sua analisi, si rivela particolarmente fecondo: partendo dal principio che saper maneggiare il verso fosse una competenza indispensabile per chiunque frequentasse gli ambienti delle corti, queste collezioni talvolta mediocri dal punto di vista strettamente letterario si rivelano uno strumento prezioso per comprendere il tipo di creatività e di piacere estetico che stanno alla base della loro produzione, il loro significato culturale in 
quanto atto sociale e le caratteristiche dell'ambiente da cui essi sono scaturiti. Una lettura dei testi che tenga conto del contesto codicologico nel quale sono inseriti e prenda in esame serie di liriche coerenti tra loro consentirà dunque di apprezzarne maggiormente il significato culturale.

La vasta materia di questa ricerca è stata suddivisa in quattro capitoli. Nel primo, l'A. parte dall'analisi delle collezioni di 'venditions d'amour', da quelle meno note contenute in un incunabolo della Bibliothèque nationale de France, a quelle più note di Christine de Pizan. Attraverso altri testi che mettono in scena la creazione poetica come evento mondano, quali il Pastoralet, sorta di romanzo a chiave di ambiente borgognone, o il Parfait du Paon di Jean de la Mote, ma anche il corposo volume dell'opera di Eustache Deschamps (BnF, ms. fr. 840), J. Taylor mostra che i giochi sociali fondati sulla produzione di brevi liriche coinvolgevano anche i poeti professionisti e possono considerarsi espressione delle abitudini delle diverse gerarchie intellettuali, le quali utilizzavano all'interno della corte il 'capitale culturale' che ne derivava. È chiara qui l'eco delle teorie sociologiche di Pierre Bourdieu, che ispirano esplicitamente questo studio insieme al concetto di contenuto metacomunicativo, mutuato dalle teorie dell'antropologo Geoffrey Bateson: il libro in quanto oggetto è considerato cioè essenzialmente come risultato della relazione tra esseri umani e, di conseguenza, le raccolte prese in esame hanno soprattutto un valore documentario per lo studio dei rapporti sociali che li hanno prodotti. Ne sono un esempio i manoscritti BnF, fr. $1719 \mathrm{e}$ 217 della Bibliothèque Intercommunale Épinal-Golbey, considerati molto opportunamente 'eventi testuali', perché il loro interesse sta nel processo che è alla base della costruzione del loro contenuto, più che nella qualità delle liriche raccolte in essi.

In un'ottica simile viene esaminato anche il manoscritto personale di Charles d'Orléans, che costituisce l'oggetto del secondo capitolo. L'attenzione si focalizza in particolare sul passaggio del codice da antologia personale a raccolta miscellanea, diventando luogo ed occasione di dialogo poetico: le liriche del duca diventano fonte di ispirazione per versificatori più o meno occasionali, mentre la dinamica della raccolta assume un aspetto plurivocale, recando la traccia di differenti pratiche poetiche e sociali. Tra le varie sequenze di componimenti esaminati, figurano lo scambio poetico tra il duca, Fredet, Simonet Caillau e Jean de Lorraine, quello con Villon e quello con René d'Anjou, oltre alla sequenza di rondeaux costruiti intorno al verso 'Jaulier des prisons de Pensée', che mostrano una profonda coerenza ed unità narrativa.

Il capitolo successivo è incentrato sul manoscritto Carpentras, Bibliothèque Inguimbertine, 375, appartenuto a Marie de Clèves duchessa d'Orléans, e costituito in gran parte da copie delle liriche del duca. Soffermandosi sui componimenti non presenti nel manoscritto personale di Charles, l'A. dimostra che il codice di Carpentras possiede un'identità poetica propria, la quale permette forse anche di rivedere i giudizi critici sulla personalità di Marie. Il funzionamento dei testi riuniti in questo manoscritto 'di coterie' è messo in parallelo con quello di altri due codici, correlati tra loro e legati in qualche modo alla corte di Blois (Paris, BnF, n.a.f. 15771 e fr. 9223). Dopo aver fornito una tavola dettagliata del loro contenuto, J. Taylor mostra che essi possono essere considerati «evidence of the malleability of the late-medieval lyric, and they suggest ways in which the late-medieval poetic miscellany is responsive to new or personal recombinations and reinterpretations» (p. 186). Per dimostrarlo, la studiosa analizza in primo luogo la questione degli autori che vi hanno contribuito, in relazione 
alla loro presenza in altre raccolte antologiche o miscellanee; in seguito, viene preso in esame il procedimento con cui vengono generati alcuni gruppi di liriche a partire da temi e versi chiave, che paiono circolare di corte in corte. Da ultimo, lo studio dell'album poetico di Margherita d'Austria permette di esaminare la dinamica e la fisionomia particolari di alcuni manoscritti 'di coterie'.

La raccolta poetica a stampa pubblicata da Antoine Vérard nel 1501, che occupa il capitolo conclusivo di questo lavoro, condivide con questi ultimi la volontà di fare dell'antologia la testimonianza di un dialogo incentrato su una finzione di intimità. Impresa rivoluzionaria, che inserisce un ingente numero di liriche eterogenee all'interno di un elaborato contesto narrativo, questo volume è però destinato ad un pubblico borghese, e perciò molto diverso rispetto alle antologie e miscellanee esaminate fin qui. L'A. analizza dapprima il paratesto, anche iconografico, che permette di strutturare il materiale attraverso la forma del dialogo tra personaggi; espressione della volontà di sottolineare l'impressione di controllo editoriale sulla materia, questa complessa struttura permette di organizzare le oltre seicento liriche che compongono il volume. Altrettanto rivelatrice delle intenzioni che sottendono questa impresa editoriale è la breve Ars poetica che apre la raccolta, la cui funzione è, secondo l'A., di fornire ad un pubblico non così avvezzo alle tecniche di versificazione come quello dei cortigiani uno strumento pratico per apprezzare il contenuto della raccolta: strategia ingegnosa di un editore intraprendente, in cerca di nuovi mercati. Infatti, la presenza di temi e motivi poetici ricorrenti permette di fornire al lettore spunti per partecipare attivamente alla ricerca di un significato e di una coerenza, nel tentativo di accrescere in lui il piacere della lettura. È quanto accade per il gruppo di liriche che ruotano intorno alla Ballade des pendus di Villon.

Questo studio denso e bene informato contribuisce efficacemente ad accrescere la conoscenza intorno ai meccanismi di produzione della poesia lirica tardomedievale, fornendo un utile complemento agli studi fondatori in questo campo, anche sotto il profilo della documentazione bibliografica, i cui rinvii avrebbero tuttavia potuto essere riassunti in un'apposita sezione alla fine del volume per facilitarne l'identificazione. 